Research Article

\title{
Finite Element Modeling of Stress Behavior of FGM Nanoplates
}

\author{
Nguyen Thi Giang \\ University of Transport Technology, 54 Trieu Khuc, Thanh Xuan, Hanoi, Vietnam \\ Correspondence should be addressed to Nguyen Thi Giang; giangnt@utt.edu.vn \\ Received 28 March 2021; Revised 4 May 2021; Accepted 18 May 2021; Published 27 May 2021 \\ Academic Editor: Hamed Akhavan \\ Copyright (C) 2021 Nguyen Thi Giang. This is an open access article distributed under the Creative Commons Attribution License, \\ which permits unrestricted use, distribution, and reproduction in any medium, provided the original work is properly cited. \\ The mechanical response investigation of nanoplates especially the stress distribution plays a very important role in engineering \\ practice, which is a condition to help test the durability as well as design and use the nanoplate structures most effectively. This \\ pioneering paper uses the finite element method to simulate the stress field of FGM nanoplates based on the first-order shear \\ deformation theory of Mindlin. The finite element formulations are derived by taking into account the effect of the nonlocal \\ coefficient to analyze the mechanical response of nanometer-scale plates. This work presents the distribution of stress components \\ in the $x y$-plane of plates with different boundary conditions. The numerical results also show clearly that the nonlocal coefficient \\ has a significant influence on the deflection and stress of FGM nanoplates. These numerical results are very new and stunning \\ which clearly show the position of the stress reaching the maximum value. This work is also the basis for scientists in testing the \\ durability of FGM nanoplates.
}

\section{Introduction}

Functionally graded materials (FGMs) have been widely used in technical practice. These are a new type of material with many outstanding advantages such as good bearing capacity, abrasion resistance, and good working in a hightemperature environment. The reason given is that this type of material is made up of two or more component materials, mainly ceramics and metals, so FGMs carry the full range of features of ceramics and metals. Due to the wide applicability of FGM structures, scientists have been focusing on the mechanical behavior of structures made of such materials. Bui et al. [1] used the finite element method to analyze the static bending and free vibration of FGM plates in a thermal environment. For the static problem, the author only considered the stress distribution at the midpoint of FGM plates. Demirbas [2] used the three-dimensional elasticity theory to study the transient thermal residual stress analyses of onedimensional FGM plates, in which the plate was clamped at all edges. Adineh and Kadkhodayan [3] studied the threedimensional thermo-mechanical analysis of a 3D-FGM skew plate on an elastic foundation, where the stress response of structures was investigated, but only considering the stress distribution along with the plate thickness at the center point and the corner point of plates. Do and his coworkers [4] used the third-order shear deformation theory and the finite element method to analyze the mechanical behavior of $2 \mathrm{D}$ FGM plates; however, the authors just investigated the deflection and stress at one point of plates. Yahia et al. [5] explored the effect of homogenization models on stress analysis of functionally graded plates, where the stress distribution along the plate thickness was figured out by using the analytical solution, and only fully simply supported plates were explored.

Nowadays, with the development of science and technology, nanoscale structures made of FGM materials are used more and more in practice such as biological building blocks, solar cells, artificial structures, micro/nanosensors, micro/nano-electro-mechanical systems (MEMS and NEMS). The use of nanostructures is increasing in the exponential principle, so they have attracted a lot of researchers' attention. However, because of its nanosize, the calculations for FGM nanostructures are much different from the traditional structures; therefore, normally one has to take into account the effect of small-scale effects. The computation to determine bending behavior, as well as stress analysis of FGM nanoplates, has yielded some preliminary results. Hosseini et al. [6] researched the stress analysis of 
rotating nanodisk made of functionally graded materials with variable thickness, and the results found out that the effects of thickness parameters are greater than the effect of graded-index. Shahriar and Akgöz [7] employed three-dimensional elasticity theory to investigate the static and dynamic behaviors of FGM nanoplates, and the authors focused on the variation of deflection along with the thickness of this structure. Ansari et al. [8] presented the bending analysis of nanoplates resting on elastic foundation based on the integral formulation of Eringen's nonlocal theory, where the authors were only interested in the maximum deflection according to the force effect direction for nanoplates with different boundary conditions. Babu and Patel [9] used the classical plate theory of Kirchhoff to explore the static bending, free vibration, and buckling of nanoplates with different boundary conditions, in which the static bending problem only gave the maximum deflection result of the plate under static load. Repka et al. [10] explored the bending of plates subjected to stationary transversal loading based on a moving finite elements method, and the results focused on vertical deflection $w$ in the direction perpendicular to the plane of plates. Thai et al. [11] used modified isogeometric analysis (IGA) to analyze the free vibration and bending of nano FGM plates, where the calculation results for the static bending problem just stopped at the static deflection and did not mention the stresses. Ansari et al. [12] researched nonlinear static bending of functionally graded grapheneplatelet reinforced composite porous plates with arbitrary shape, where the survey results did not mention the stresses of this plate. Recently, there are some works [13-18] based on nonlocal elasticity theory to investigate the buckling and vibration responses of the nanostructures. And the works [19-21] flexibly used a variety of beam and plate theories to study bending, buckling, and dynamic response of the FGM structures.

Through the published data, one can see that most of these works mainly went into the stress and displacement behavior analysis of some points in the plate, usually the midpoint of the plate for plates with symmetrical boundary such as fully simply supported and fully clamped supported conditions. This makes it difficult to consider the overall stress distribution for the entire structure. However, from the stress distribution of the entire plate, it is of great help to test the strength and evaluate the failure position for the nanoplates as the basis for the design calculation and the most efficient use. Therefore, this work is the different and newest point compared with other published investigations. This work uses the finite element method to simulate the stress field of FGM nanoplates subjected to static loads. The finite element formulations are established based on the firstorder shear deformation theory of Mindlin taking into consideration of small-scale effects. The numerical results show that the stress components reach maximum at different positions and depending on the boundary conditions of the plate, and these are new simulation results, which will greatly help in the work of evaluating the bearing capacity of FGM nanostructures.
The body of this work is as follows: Section 2 gives a finite element solution for the bending problem of FGM nanoplates. Continuously, Section 3 reveals the verification study to show the accuracy and convergence of the proposed theory. Section 4 indicates the numerical and graphical investigation of FGM nanoplates, especially the stress field of FGM nanoplates. Finally, Section 5 summarizes some important conclusions on the numerical results of this work.

\section{Finite Element Model of FGM Nanoplates in Static Bending Problem}

Consider a rectangular FGM nanoplate (Figure 1) with length $a$, width $b$, and thickness $h$. The material properties are defined as follows $[1,22-24]$ :

$$
R(z)=R_{m}+\left(R_{c}-R_{m}\right) V_{c},
$$

with $R_{c}$ and $R_{m}$ denote Young's modulus and Poisson's ratio of ceramic and metal, respectively. The volume portions of ceramic $\left(V_{c}\right)$ and metal $\left(V_{m}\right)$ are calculated as follows $[1,22]$ :

$$
\begin{aligned}
V_{m}+V_{c} & =1, \\
V_{c} & =\left(\frac{1}{2}+\frac{z}{h}\right)^{n},
\end{aligned}
$$

where $n$ is the volume fraction exponent.

\section{Nonlocal Elasticity Theory}

Based on the nonlocal theory of Eringen, the nonlocal stress tensor at any point [25]

$$
\sigma_{i j}=\int_{V} \alpha\left(\left|x^{\prime}-\mathbf{x}\right|\right) \sigma_{i j}^{L} \mathrm{~d} V\left(x^{\prime}\right),
$$

in which the Hookean stress tensor is calculated as

$$
\sigma_{i j}^{L}=c_{i j k l} \varepsilon_{k l}
$$

and $\alpha\left(\left|x^{\prime}-\mathbf{x}\right|\right)$ is the kernel function which is normalized over the whole body. This function can be obtained by combining the lattice dynamics with nonlocal results [25]. For instance, the kernel function for $2 \mathrm{D}$ problems has the following form:

$$
\alpha(|\mathbf{x}|)=\left(2 \pi l^{2} \vartheta^{2}\right)^{-1} H_{0}\left(\frac{|\mathbf{x}|}{l \vartheta}\right), \quad \vartheta=\frac{e_{0} a}{l},
$$

where $H_{0}$ is the improved Bessel function, $a$ and $l$ are internal and external characteristic lengths, respectively, and $e_{0}$ is material constant, which is found by the experiment. On the other hand, nonlocal elasticity is related to spatial integrals that present weighted averages of the contributions of the strain component of all points in the continuum body to the stress tensor at a point.

In the nonlocal linear elasticity, the equation of motion can be obtained from nonlocal balance law as follows: 


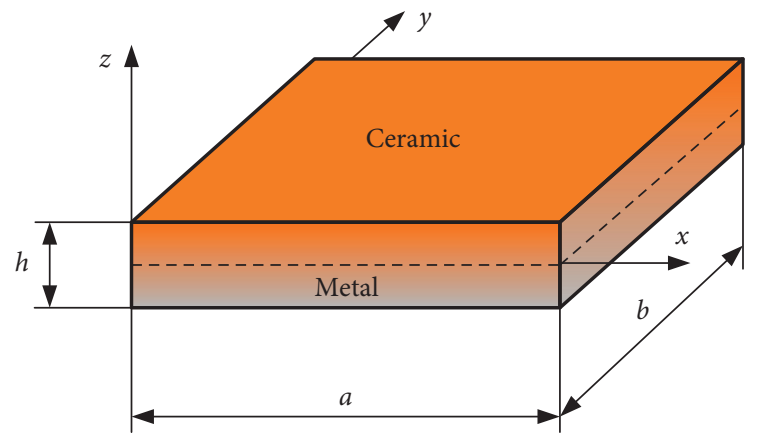

FIGURE 1: A model of an FGM nanoplate.

$$
\sigma_{i j, j}+f_{i}=0
$$

in which $i$ and $j$ take the symbols $x, y$, and $z$ and $f_{i}$ is the parts of the body load [25]. Substituting equation (3) into equation (6), the integral form of nonlocal constitutive equation is possessed. It is seen that solving an integral equation is more complicated than a differential equation; therefore, Eringen $[25,26]$ proposed a differential form of the nonlocal constitutive equation as follows:

$$
\sigma_{i j, j}+\Theta\left(f_{i}\right)=0
$$

where the linear differential operator $\Theta$ is calculated as

$$
\Theta=\left[1-\psi^{2} \nabla^{2}\right]
$$

in which $\nabla^{2}=\left(\partial^{2} / \partial x^{2}\right)+\left(\partial^{2} / \partial y^{2}\right)$ is the Laplace operator, and $\psi^{2}\left(n m^{2}\right)=\left(e_{0} a\right)^{2}$ is the nonlocal coefficient, where $a$ is an internal characteristic length and $e_{0}$ is a constant material, which is found by the experiment.
By substituting this operator into equation (3), the constitutive equation can be obtained as follows:

$$
\left[1-\psi^{2} \nabla^{2}\right] \sigma=C \varepsilon
$$

In the general case, the nonlocality is naturally threedimensional, rather than the two-dimensional simplification currently in nanostructures $[27,28]$. When the feature size of plates is reduced to the nanoscale, classical mechanics will break down firstly at the thickness direction of plates due to the simple fact that plates' thickness dimensions are far smaller than their length dimension. This in turn implies that the nonlocal effect in the thickness is likely to play a dominant role in the contribution of size dependence and the cross section effect on the nonlocal stress, the bending moment, and the deflection [27]. Equation (9) is just an approximation; however, it is simpler and more convenient than the integral relation (3) to apply to various linear elasticity problems.

According to the first-order shear deformation theory of Mindlin, the displacement field at any points within the plate is expressed as follows $[8,29]$ :

$$
\left\{\begin{array}{l}
u(x, y, z)=u_{0}(x, y, 0)+z \phi_{x}, \\
v(x, y, z)=v_{0}(x, y, 0)+z \phi_{y}, \\
w(x, y, z)=w_{0}(x, y, 0),
\end{array}\right.
$$

in which $u, v$, and $w$ are the displacements along the $x$-, $y$-, and $z$-directions; $u_{0}, v_{0}$, and $w_{0}$ are the in-plane displacements at that point where $z=0$; and $\phi_{x}$ and $\phi_{y}$ are the rotations around $O y$ and $O x$, respectively.

The strain components are calculated through the derivatives as follows:

$$
\left\{\begin{array}{l}
\boldsymbol{\varepsilon}=\left\{\begin{array}{c}
\varepsilon_{x x} \\
\varepsilon_{y y} \\
\gamma_{x y}
\end{array}\right\}=\left\{\begin{array}{c}
\frac{\partial u_{0}}{\partial x}+z \frac{\partial \phi_{x}}{\partial x}=\varepsilon_{x x}^{0}+z \kappa_{x x} \\
\frac{\partial v_{0}}{\partial y}+z \frac{\partial \phi_{y}}{\partial y}=\varepsilon_{y y}^{0}+z \kappa_{y y} \\
\frac{\partial u_{0}}{\partial y}+\frac{\partial v_{0}}{\partial x}+z\left(\frac{\partial \phi_{x}}{\partial y}+\frac{\partial \phi_{x}}{\partial y}\right)=\gamma_{x y}^{0}+z \kappa_{x y}
\end{array}\right\}=\left\{\begin{array}{c}
\varepsilon_{x x}^{0} \\
\left.\varepsilon_{y y}^{0}\right\}+z\left\{\begin{array}{c}
\kappa_{x x} \\
\left.\kappa_{y y}\right\} \\
\gamma_{x y}^{0}
\end{array}\right\} \\
\varepsilon_{0} \\
\gamma_{x z} \\
\kappa_{x y}
\end{array}\right\}=\left\{\begin{array}{l}
\frac{\partial w}{\partial x}+\phi_{x} \\
\frac{\partial w}{\partial y}+\phi_{y}
\end{array}\right\} .
\end{array}\right.
$$

At this point, equation (9) is specifically rewritten in the following form: 


$$
\begin{aligned}
& {\left[1-\psi^{2} \nabla^{2}\right] \sigma_{x x}=\frac{E(z)}{1-\nu(z)^{2}}\left[\frac{\partial u_{0}}{\partial x}+z \frac{\partial \phi_{x}}{\partial x}+v(z)\left(\frac{\partial u_{0}}{\partial x}+z \frac{\partial \phi_{x}}{\partial x}\right)\right]} \\
& {\left[1-\psi^{2} \nabla^{2}\right] \sigma_{y y}=\frac{E(z)}{1-\nu(z)^{2}}\left[\frac{\partial v_{0}}{\partial y}+z \frac{\partial \phi_{y}}{\partial y}+v(z)\left(\frac{\partial v_{0}}{\partial y}+z \frac{\partial \phi_{y}}{\partial y}\right)\right]} \\
& {\left[1-\psi^{2} \nabla^{2}\right] \tau_{x y}=\frac{E(z)}{2(1+\nu(z))}\left(\frac{\partial u_{0}}{\partial y}+\frac{\partial v_{0}}{\partial x}+z\left(\frac{\partial \phi_{x}}{\partial y}+\frac{\partial \phi_{x}}{\partial y}\right)\right)} \\
& {\left[1-\psi^{2} \nabla^{2}\right] \tau_{y z}=\frac{E(z)}{2(1+v(z))}\left(\frac{\partial w}{\partial x}+\phi_{x}\right)} \\
& {\left[1-\psi^{2} \nabla^{2}\right] \tau_{x z}=\frac{E(z)}{2(1+\nu(z))}\left(\frac{\partial w}{\partial y}+\phi_{y}\right)}
\end{aligned}
$$

The nonlocal normal forces, moments, and shear forces are calculated through integrals as follows:

$$
\begin{aligned}
& {\left[1-\psi^{2} \nabla^{2}\right]\left\{\begin{array}{c}
N_{x x} \\
N_{y y} \\
N_{x y}
\end{array}\right\}=\int_{-(h / 2)}^{h / 2}\left[1-\psi^{2} \nabla^{2}\right]\left\{\begin{array}{c}
\sigma_{x x} \\
\sigma_{y y} \\
\tau_{x y}
\end{array}\right\} \mathrm{d} z,} \\
& {\left[1-\psi^{2} \nabla^{2}\right]\left\{\begin{array}{c}
M_{x x} \\
M_{y y} \\
M_{x y}
\end{array}\right\}=\int_{-(h / 2)}^{h / 2} z\left[1-\psi^{2} \nabla^{2}\right]\left\{\begin{array}{c}
\sigma_{x x} \\
\sigma_{y y} \\
\tau_{x y}
\end{array}\right\} \mathrm{d} z,} \\
& {\left[1-\psi^{2} \nabla^{2}\right]\left\{\begin{array}{c}
Q_{x z} \\
Q_{y z}
\end{array}\right\}=\int_{-(h / 2)}^{h / 2}\left[1-\psi^{2} \nabla^{2}\right]\left\{\begin{array}{c}
\tau_{x z} \\
\tau_{y z}
\end{array}\right\} \mathrm{d} z .}
\end{aligned}
$$

$$
\begin{aligned}
{\left[1-\psi^{2} \nabla^{2}\right] N_{x x} } & =\int_{-(h / 2)}^{h / 2} \frac{E(z)}{1-v(z)^{2}}\left[\frac{\partial u_{0}}{\partial x}+z \frac{\partial \phi_{x}}{\partial x}+v(z)\left(\frac{\partial u_{0}}{\partial x}+z \frac{\partial \phi_{x}}{\partial x}\right)\right] \mathrm{d} z \\
{\left[1-\psi^{2} \nabla^{2}\right] N_{y y} } & =\int_{-(h / 2)}^{h / 2} \frac{E(z)}{1-v(z)^{2}}\left[\frac{\partial v_{0}}{\partial y}+z \frac{\partial \phi_{y}}{\partial y}+v(z)\left(\frac{\partial v_{0}}{\partial y}+z \frac{\partial \phi_{y}}{\partial y}\right)\right] \mathrm{d} z \\
{\left[1-\psi^{2} \nabla^{2}\right] N_{x y} } & =\int_{-(h / 2)}^{h / 2} \frac{E(z)}{\left(1-v(z)^{2}\right)}\left[\frac{\partial u_{0}}{\partial y}+\frac{\partial v_{0}}{\partial x}+z\left(\frac{\partial \phi_{x}}{\partial y}+\frac{\partial \phi_{x}}{\partial y}\right)\right] \mathrm{d} z \\
{\left[1-\psi^{2} \nabla^{2}\right] M_{x x} } & =\int_{-(h / 2)}^{h / 2} \frac{z E(z)}{1-v(z)^{2}}\left[\frac{\partial u_{0}}{\partial x}+z \frac{\partial \phi_{x}}{\partial x}+v(z)\left(\frac{\partial u_{0}}{\partial x}+z \frac{\partial \phi_{x}}{\partial x}\right)\right] \mathrm{d} z, \\
{\left[1-\psi^{2} \nabla^{2}\right] M_{y y} } & =\int_{-(h / 2)}^{h / 2} \frac{z E(z)}{1-v(z)^{2}}\left[\frac{\partial v_{0}}{\partial y}+z \frac{\partial \phi_{y}}{\partial y}+v(z)\left(\frac{\partial v_{0}}{\partial y}+z \frac{\partial \phi_{y}}{\partial y}\right)\right] \mathrm{d} z, \\
{\left[1-\psi^{2} \nabla^{2}\right] M_{x y} } & =\int_{-(h / 2)}^{h / 2} \frac{z E(z)}{\left(1-v(z)^{2}\right)}\left[\frac{\partial u_{0}}{\partial y}+\frac{\partial v_{0}}{\partial x}+z\left(\frac{\partial \phi_{x}}{\partial y}+\frac{\partial \phi_{x}}{\partial y}\right)\right] \mathrm{d} z, \\
{\left[1-\psi^{2} \nabla^{2}\right] Q_{x z} } & =\int_{-(h / 2)}^{h / 2} \frac{E(z)}{2(1+v(z))}\left(\frac{\partial w}{\partial x}+\phi_{x}\right) \mathrm{d} z \\
{\left[1-\psi^{2} \nabla^{2}\right] Q_{y z} } & =\int_{-(h / 2)}^{h / 2} \frac{E(z)}{2(1+v(z))}\left(\frac{\partial w}{\partial y}+\phi_{y}\right) \mathrm{d} z .
\end{aligned}
$$


Equations (15)-(17) can be written in the matrix form as follows:

$$
\begin{aligned}
& \left\{\begin{array}{l}
{\left[1-\psi^{2} \nabla^{2}\right] N_{x x}} \\
\left.\left[1-\psi^{2} \nabla^{2}\right] N_{y y}\right\} \\
{\left[1-\psi^{2} \nabla^{2}\right] N_{x y}}
\end{array}\right\}=\mathbf{A}\left\{\begin{array}{c}
\varepsilon_{x x}^{0} \\
\varepsilon_{y y}^{0} \\
\gamma_{x y}^{0}
\end{array}\right\}+\mathbf{B}\left\{\begin{array}{c}
\kappa_{x x} \\
\kappa_{y y} \\
\kappa_{x y}
\end{array}\right\}, \\
& \left\{\begin{array}{l}
{\left[1-\psi^{2} \nabla^{2}\right] M_{x x}} \\
{\left[1-\psi^{2} \nabla^{2}\right] M_{y y}} \\
{\left[1-\psi^{2} \nabla^{2}\right] M_{x y}}
\end{array}\right\}=\mathbf{B}\left\{\begin{array}{c}
\varepsilon_{x x}^{0} \\
\varepsilon_{y y}^{0} \\
\gamma_{x y}^{0}
\end{array}\right\}+\mathbf{D}\left\{\begin{array}{c}
\kappa_{x x} \\
\kappa_{y y} \\
\kappa_{x y}
\end{array}\right\}, \\
& \left\{\begin{array}{l}
{\left[1-\psi^{2} \nabla^{2}\right] Q_{x z}} \\
{\left[1-\psi^{2} \nabla^{2}\right] Q_{y z}}
\end{array}\right\}=\mathbf{D}_{s}\left\{\begin{array}{c}
\gamma_{x z} \\
\gamma_{y z}
\end{array}\right\},
\end{aligned}
$$
[30]:

$$
\begin{aligned}
\{\mathbf{A}, \mathbf{B}, \mathbf{D}\} & =\int_{-(h / 2)}^{h / 2} \frac{E(z)}{1-v(z)^{2}}\left[\begin{array}{ccc}
1 & \nu(z) & 0 \\
\nu(z) & 1 & 0 \\
0 & 0 & \frac{1+\nu(z)}{2}
\end{array}\right]\left\{1, z, z^{2}\right\} \mathrm{d} z \\
\left\{\mathbf{D}_{s}\right\} & =\int_{-(h / 2)}^{h / 2} \frac{E(z)}{2(1+\nu(z))}\left[\begin{array}{ll}
1 & 0 \\
0 & 1
\end{array}\right] \mathrm{d} z .
\end{aligned}
$$

The strain energy of the nanoplate has the following form

$$
\Pi=\frac{1}{2} \int_{V} \sigma_{i j} \varepsilon_{i j} \mathrm{~d} V .
$$

Substituting the stress and strain expressions into equation (20), one gets the following:

where

$$
\Pi=\frac{1}{2} \int_{S}\left(\begin{array}{c}
N_{x x} \varepsilon_{x x}^{0}+N_{y y} \varepsilon_{y y}^{0}+N_{x y} \gamma_{x y}^{0}+M_{x x} \kappa_{x x}+M_{y y} \kappa_{y y}+M_{x y} \kappa_{x y} \\
+Q_{x z} \gamma_{x z}+Q_{y z} \gamma_{y z}
\end{array}\right) \mathrm{d} S .
$$

The variation of equation (21) has the following form:

$$
\begin{array}{r}
\delta \Pi=\int_{S}\left(\begin{array}{c}
N_{x x} \delta \varepsilon_{x x}^{0}+N_{y y} \delta \varepsilon_{y y}^{0}+N_{x y} \delta \gamma_{x y}^{0}+\delta M_{x x} \delta \kappa_{x x}+M_{y y} \delta \kappa_{y y}+M_{x y} \delta \kappa_{x y} \\
+Q_{x z} \delta \gamma_{x z}+Q_{y z} \delta \gamma_{y z}
\end{array}\right) \mathrm{d} S-\int_{S}(q \delta w) \mathrm{d} S \\
=\int_{S}\left(\begin{array}{c}
N_{x x} \delta\left(\frac{\partial u_{0}}{\partial x}\right)+N_{y y} \delta\left(\frac{\partial v_{0}}{\partial y}\right)+N_{x y} \delta\left(\frac{\partial v_{0}}{\partial x}+\frac{\partial u_{0}}{\partial y}\right) \\
+\delta M_{x x} \delta\left(\frac{\partial \phi_{x}}{\partial x}\right)+M_{y y} \delta\left(\frac{\partial \phi_{y}}{\partial y}\right)+M_{x y} \delta\left(\frac{\partial \phi_{x}}{\partial y}+\frac{\partial \phi_{y}}{\partial x}\right) \\
+Q_{x z} \delta\left(\frac{\partial w}{\partial x}+\phi_{x}\right)+Q_{y z} \delta\left(\frac{\partial w}{\partial y}+\phi_{y}\right)
\end{array}\right) \mathrm{d} S-(q \delta w) \mathrm{d} S,
\end{array}
$$

in which $q$ is the transverse distributed force.

After performing partial integral and setting the coefficients of $\delta u_{0}, \delta v_{0}, \delta \phi_{x}, \delta \phi_{y}$, and $\delta w$ equal to zero, one gets the following: 


$$
\begin{gathered}
\delta u_{0}: \frac{\partial N_{x x}}{\partial x}+\frac{\partial N_{x y}}{\partial y}=0 \\
\delta v_{0}: \frac{\partial N_{y}}{\partial y}+\frac{\partial N_{x y}}{\partial x}=0 \\
\delta \phi_{x}: \frac{\partial M_{x x}}{\partial x}+\frac{\partial M_{x y}}{\partial y}-Q_{x z}=0 \\
\delta \phi_{y}: \frac{\partial M_{y y}}{\partial y}+\frac{\partial M_{x y}}{\partial x}-Q_{y z}=0 \\
\delta w: \frac{\partial Q_{x z}}{\partial x}+\frac{\partial Q_{y z}}{\partial y}=q .
\end{gathered}
$$

Substituting equations (18)-(20) into equations (23)-(25), we have the following expressions:

$$
\begin{gathered}
\int_{S}\left(N_{x x} \delta\left(\frac{\partial u_{0}}{\partial x}\right)+N_{x y} \delta\left(\frac{\partial u_{0}}{\partial y}\right)\right) \mathrm{d} S=0 \\
\int_{S}\left(N_{y y} \delta\left(\frac{\partial v_{0}}{\partial y}\right)+N_{x y} \delta\left(\frac{\partial v_{0}}{\partial x}\right)\right) \mathrm{d} S=0 \\
\int_{S}\left(M_{x x} \delta\left(\frac{\partial \phi_{x}}{\partial x}\right)+M_{x y} \delta\left(\frac{\partial \phi_{x}}{\partial y}+\frac{\partial \phi_{y}}{\partial y}\right)\right. \\
\left.+M_{y y} \delta\left(\frac{\partial \phi_{y}}{\partial y}\right)-Q_{x z} \delta \phi_{x}-Q_{y z} \delta \phi_{y}\right)=0 \\
\int_{S}\left(Q_{x z} \delta\left(\frac{\partial w}{\partial y}\right)+Q_{y z} \delta\left(\frac{\partial w}{\partial x}\right)-\Theta q\right) \mathrm{d} S
\end{gathered}
$$

In this work, an $m$-node plate element is used, and each node has five degrees of freedom:

$$
\mathbf{u}_{e}=\sum^{m}\left\{u_{0 i}, v_{0 i}, w_{i}, \phi_{x i}, \phi_{y i}\right\}^{T} \text {. }
$$

Then, we obtain

$$
\left\{u_{0}=\sum_{i=1}^{m} \mathbf{N}_{u} \mathbf{u}_{e}, v_{0}=\sum_{i=1}^{m} \mathbf{N}_{v} \mathbf{u}_{e}, w=\sum_{i=1}^{m} \mathbf{N}_{w} \mathbf{u}_{e}, \phi_{x}=\sum_{i=1}^{m} \mathbf{N}_{\phi_{y}} \mathbf{u}_{e}, \phi_{y}=\sum_{i=1}^{m} \mathbf{N}_{\phi_{y}} \mathbf{u}_{e} .\right.
$$

Substituting the expressions in equation (28) into equations (25)-(28), the static equilibrium equation of the plate is written in the form of finite element formulation as follows:

$$
\begin{aligned}
\mathbf{K}_{e} \mathbf{u}_{e} & =\mathbf{F}_{e}, \\
\mathbf{K}_{e} & =\int_{S}\left(\mathbf{B}_{1} \mathbf{A B}_{1}+\mathbf{B}_{1} \mathbf{B} \mathbf{B}_{2}+\mathbf{B}_{2} \mathbf{B B}_{1}+\mathbf{B}_{2} \mathbf{D B} \mathbf{B}_{2}+\mathbf{B}_{3} \mathbf{D}_{s} \mathbf{B}_{3}\right) \mathrm{d} S,
\end{aligned}
$$

where

$$
\mathbf{F}_{e}=\int_{S}\left[1-\psi^{2} \nabla^{2}\right] \mathbf{N}_{w} \mathbf{q} \mathrm{d} S,
$$

with 


$$
\begin{aligned}
& \mathbf{B}_{1}=\sum_{i=1}^{m}\left[\begin{array}{c}
\frac{\partial N_{u}}{\partial x} \\
\frac{\partial N_{v}}{\partial y} \\
\frac{\partial N_{u}}{\partial y}+\frac{\partial N_{v}}{\partial x}
\end{array}\right] \\
& \mathbf{B}_{2}=\sum_{i=1}^{m}\left[\begin{array}{c}
\frac{\partial N_{\phi_{x}}}{\partial x} \\
\frac{\partial N_{\phi_{y}}}{\partial y} \\
\frac{\partial N_{\phi_{x}}}{\partial y}+\frac{\partial N_{\phi_{y}}}{\partial x}
\end{array}\right] \\
& \mathbf{B}_{3}=\sum_{i=1}^{m}\left[\begin{array}{c}
\frac{\partial N_{w}}{\partial x+N_{\phi_{x}}} \\
\frac{\partial N_{w}}{\partial y}+N_{\phi_{y}}
\end{array}\right] \\
& \mathbf{N}_{w}=\sum_{i=1}^{m}\left[N_{w}\right] .
\end{aligned}
$$

So, from equation (36), one can get displacements of the plate, and then substituting these displacements into equations (12) and (13), we obtain the stress field of FGM nanoplates.

Boundary conditions are expressed as follows:

(i) The plate is simply supported at $x=0$ and $x=a$ :

$$
\begin{gathered}
u_{0} \\
v_{0} \\
w \\
\phi_{y}
\end{gathered}
$$

(ii) The plate is simply supported $y=0$ and $y=b$ :

$$
\begin{gathered}
u_{0}=0, \\
v_{0}=0, \\
w=0, \\
\phi_{x}=0 .
\end{gathered}
$$

(iii) One edge is clamped:

$$
\begin{gathered}
u_{0}=0, \\
v_{0}=0, \\
w=0, \\
\phi_{x}=0, \\
\phi_{y}=0 .
\end{gathered}
$$

Herein, some acronyms are explained as follows: simply supported edge-S, clamped edge-C, and free edge-F. Several boundary conditions of the plate used in this paper are presented in Figure 2.

\section{Verification Study}

In this section, numerical results of displacement and stress of FGM plate and nanoplate are compared with those of published data to confirm the reliability of the calculation theory.

Example 1. Consider an FGM plate with material properties $E_{c}=380 \mathrm{GPa}, E_{m}=70 \mathrm{GPa}, \nu_{c}=\nu_{m}=0.3, a / b=1$, and $a /$ $h=10$. The plate is fully simply supported and subjected to uniformly distributed load $q_{0}$ and sinusoidal distributed load as follows:

$$
q=q_{0} \sin \left(\frac{\pi x}{a}\right) \sin \left(\frac{\pi y}{b}\right)
$$

Nondimensional displacement and stress are normalized as follows:

$$
\begin{aligned}
\widehat{w} & =\frac{10 E_{c} h^{3}}{q_{0} a^{4}} w\left(\frac{a}{2}, \frac{b}{2}, \frac{h}{2}\right), \\
\widehat{\sigma}_{x} & =\frac{10 h}{q_{0} a} \sigma_{x}\left(\frac{a}{2}, \frac{b}{2}, \frac{h}{2}\right) .
\end{aligned}
$$

Tables 1 and 2 present the results of displacement and stress of FGM plate obtained from this approach, the finite element method [31], and the analytical method [30] in which this work uses several mesh sizes, and it can be seen that with the 800-element mesh size, the accurate data can be reached. Therefore, for all upcoming investigations, this mesh is employed.

Example 2. Consider a square FGM plate with geometrical and material properties $a / b=1, a / h=10, E_{c}=380 \mathrm{GPa}$, $E_{m}=70 \mathrm{GPa}$, and $v_{c}=v_{m}=0.3$. The plate is fully simply supported and subjected to bisinusoidal load as presented in equation (35). Nondimensional stresses are calculated as the following formulas:

$$
\left\{\begin{aligned}
\left(\sigma_{x}^{* *}, \sigma_{y}^{* *}\right) & =\left(\frac{\sigma_{x}}{q_{0}}\left(\frac{a}{2}, \frac{b}{2}, z\right), \frac{\sigma_{y}}{q_{0}}\left(\frac{a}{2}, \frac{b}{2}, z\right)\right), \\
\tau_{x y}^{* *} & =\frac{\tau_{x y}(0,0, z)}{q_{0}} .
\end{aligned}\right.
$$



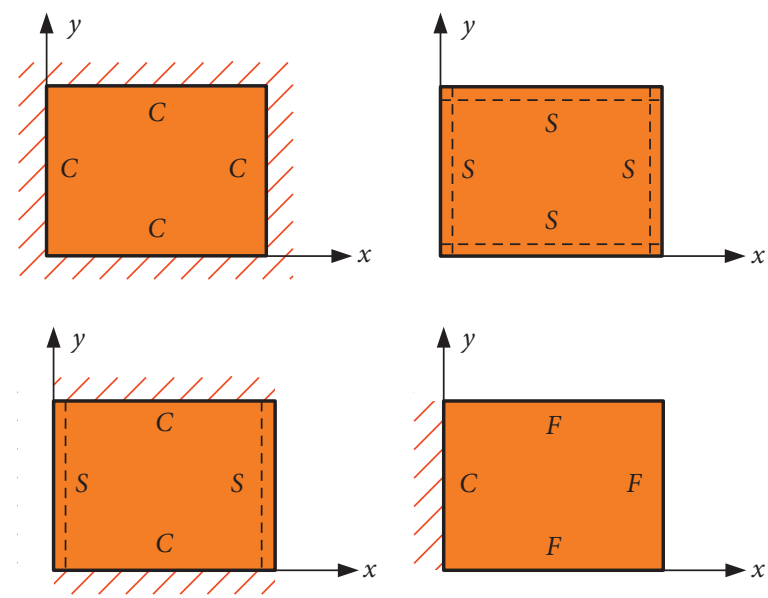

FIGURE 2: Boundary conditions of nano FGM plates.

TABLE 1: Nondimensional displacement $\widehat{w}=\left(10 E_{c} h^{3} / q_{0} a^{4}\right) w((a / 2),(b / 2))$ of square FGM plates $(a / h=10)$ subjected to uniformly distributed load and sinusoidal distributed load.

\begin{tabular}{|c|c|c|c|c|c|c|}
\hline \multirow{2}{*}{ Source } & \multicolumn{6}{|c|}{$N$} \\
\hline & & 0 & 1 & 2 & 4 & $\infty$ \\
\hline \multicolumn{7}{|c|}{ Uniform load } \\
\hline [31] & & 0.4666 & 0.9290 & 1.1952 & 1.3908 & 0.4666 \\
\hline$[30]$ & & 0.4665 & 0.9287 & 1.1940 & 1.3890 & 0.4665 \\
\hline \multirow{4}{*}{ This work } & 200 elements & 0.4666 & 0.9376 & 1.1995 & 1.3869 & 0.4666 \\
\hline & 512 elements & 0.4666 & 0.9376 & 1.1995 & 1.3869 & 0.4666 \\
\hline & 800 elements & 0.4666 & 0.9376 & 1.1995 & 1.3870 & 0.4666 \\
\hline & 1800 elements & 0.4666 & 0.9376 & 1.1995 & 1.3870 & 0.4666 \\
\hline \multicolumn{7}{|c|}{ Bisinusoidal load } \\
\hline$[31]$ & & 0.2961 & 0.5891 & 0.7582 & 0.8831 & 0.2961 \\
\hline$[30]$ & & 0.2960 & 0.5889 & 0.7573 & 0.8819 & 0.2960 \\
\hline \multirow{4}{*}{ This work } & 200 elements & 0.2960 & 0.5945 & 0.7606 & 0.8799 & 0.2960 \\
\hline & 512 elements & 0.2960 & 0.5945 & 0.7606 & 0.8800 & 0.2960 \\
\hline & 800 elements & 0.2960 & 0.5945 & 0.7606 & 0.8800 & 0.2960 \\
\hline & 1800 elements & 0.2960 & 0.5945 & 0.7606 & 0.8800 & 0.2960 \\
\hline
\end{tabular}

TABLE 2: Nondimensional stress $\widehat{\sigma}_{x}=\left(10 h / q_{0} a\right) \sigma_{x}((a / 2),(b / 2),(h / 2))$ of square FGM plates $(a / h=10)$ subjected to uniformly distributed load and sinusoidal distributed load.

\begin{tabular}{|c|c|c|c|c|c|c|}
\hline \multirow{2}{*}{ Source } & & \multicolumn{5}{|c|}{$N$} \\
\hline & & 0 & 1 & 2 & 4 & $\infty$ \\
\hline \multicolumn{7}{|c|}{ Uniform load } \\
\hline$[31]$ & & 2.8688 & 4.4303 & 5.1689 & 5.8035 & 2.8678 \\
\hline$[30]$ & & 2.8932 & 4.4745 & 5.2296 & 5.8915 & 2.8932 \\
\hline \multirow{4}{*}{ This work } & 200 elements & 2.9638 & 4.6289 & 5.4120 & 6.1146 & 2.9638 \\
\hline & 512 elements & 2.9265 & 4.5706 & 5.3438 & 6.03763 & 2.9265 \\
\hline & 800 elements & 2.9178 & 4.5570 & 5.3279 & 6.0196 & 2.9178 \\
\hline & 1800 elements & 2.9091 & 4.5435 & 5.3121 & 6.0017 & 2.9091 \\
\hline \multicolumn{7}{|c|}{ Bisinusoidal load } \\
\hline$[31]$ & & 1.9679 & 3.0389 & 3.5456 & 3.9813 & 1.9679 \\
\hline$[30]$ & & 1.9955 & 3.0870 & 3.6094 & 4.0693 & 1.9955 \\
\hline \multirow{4}{*}{ This work } & 200 elements & 2.0480 & 3.1986 & 3.7397 & 4.2252 & 0.2960 \\
\hline & 512 elements & 2.0166 & 3.1495 & 3.6823 & 4.1604 & 2.0166 \\
\hline & 800 elements & 2.0091 & 3.1379 & 3.6687 & 4.1450 & 2.0091 \\
\hline & 1800 elements & 2.0017 & 3.1262 & 3.6551 & 4.1296 & 2.0017 \\
\hline
\end{tabular}


Figure 3 presents the stress distribution by the thickness direction with different volume fraction exponent $n$ of this work and the exact solution of Hiroyuki [32]. The comparison results in Figure 3 show that the stresses are nonlinearly distributed according to the plate thickness, and these stresses reach the maximum value at the top of the plate (full of ceramic). Also from Figure 3, one can see that for the case of $n=0$ (homogeneous material), the stress is linearly distributed. However, when $n=1$, the material properties vary smoothly from one surface to another one of the plates, so the stress also varies smoothly from one side to the other side of the plate following a nonlinear curve. This represents a complete difference from homogeneous materials and laminated composites.

Example 3. Finally, the comparison of the maximum deflection of a nanoplate is presented, and this plate has al $b=1, a / h=10$, Young's modulus $E=30 \mathrm{GPa}$, and Poisson's ratio $v=0.3$. The plate is fully simply supported and subjected to uniformly distributed load $q_{0}$. The nondimensional deflection is defined as follows:

$$
\bar{w}=w_{c} \frac{1000 E_{c} h^{3}}{12\left(1-v^{2}\right) q_{0} a^{4}} .
$$

The numerical results of this work compared with those of analytical solution [33] are listed in Table 3. It can be seen that the data have a good agreement with two $b / a$ ratios.

Through all the comparison examples above, it can be seen that the established theory has the necessary accuracy.

\section{Numerical Results}

In this section, an FMG nanoplate is considered, in which the dimensions are length $a$, width $b$, thickness $h$, and mechanical properties as follows:

Ceramic $\mathrm{ZrO}_{2}: E_{c}=168 \mathrm{GPa}$ and $\nu_{c}=0.3$

Metal Al: $E_{m}=70 \mathrm{GPa}$ and $v_{m}=0.3$

As Example 2 in the above section has considered, for the plate with the mechanical property varying in the thickness direction, the maximum stress position would have the coordinate $z=h / 2$, so the following investigations focus on the analysis of stress components at this position, and these are completely novel explorations, which have never mentioned by any previous studies.

The plate is subjected to uniformly distributed load $q_{0}$ and bisinusoidal load as shown in equation (35), where the survey quantities are expressed as follows:

$$
\begin{aligned}
w^{*} & =w \frac{100 E_{c} h^{3}}{q_{0} a^{4}}, \\
\sigma_{x}^{*} & =\frac{10 h}{q_{0} a} \sigma_{x}\left(x, y, \frac{h}{2}\right), \\
\sigma_{y}^{*} & =\frac{10 h}{q_{0} a} \sigma_{y}\left(x, y, \frac{h}{2}\right), \\
\tau_{x y}^{*} & =\frac{10 h}{q_{0} a} \tau_{x y}\left(x, y, \frac{h}{2}\right), \\
\tau_{x z}^{*} & =\frac{10 h}{q_{0} a} \tau_{x z}\left(x, y, \frac{h}{2}\right) .
\end{aligned}
$$

The boundary conditions are described as follows:

(i) Fully simply supported plate: SSSS

(ii) Fully clamped plate: CCCC

(iii) The plate with two opposite edges are clamped and the other edges are free: CFCF

(iv) The plate with one edge is clamped and the other edges are free: $\mathrm{CFFF}$

5.1. Effect of Boundary Conditions on the Stress Distribution of FGM Nanoplate. In order to clearly see the positions with maximum values of stress components, this work calculates and draws the stress distribution surface of FGM nanoplate in $(x, y)$ coordinates with different boundary conditions. The numerical results are presented in Figures 4-6 and Tables 4 and 5.

From the numerical data, some discussions are given as follows:

(i) The stresses $\sigma_{x}^{*}$ and $\sigma_{y}^{*}$ obtain the maximum values at the center point of the SSSS plate and zero at the boundaries. In contrast, for the CCCC plate, these stresses obtain the maximum values at the boundaries and then they decrease gradually to zero and increase at the center point. Thus, the values of these stresses are nonzero and relatively large at the center of the plate.

(ii) The stresses $\tau_{x y}^{*}, \tau_{x z}^{*}$, and $\tau_{y z}^{*}$ have symmetrical values across the lines $x=a / 2$ and $y=b / 2$ and get zero values in the center point of the plate. The maximum values of the stress $\tau_{x y}^{*}$ are located in four corners of the SSSS plate, while they are situated in some distance from the boundary. For both SSSS and CCCC plates, the maximum values of the stresses $\tau_{x z}^{*}$ and $\tau_{y z}^{*}$ are located in the middle point of each edge. The magnitudes of the maximum values $\tau_{x z}^{*}$ and $\tau_{y z}^{*}$ for both plates are not as 


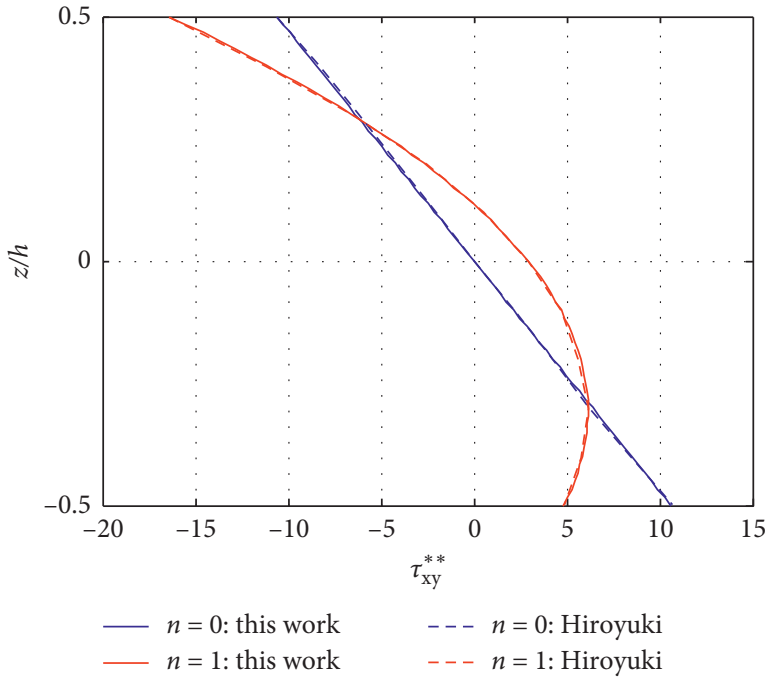

(a)

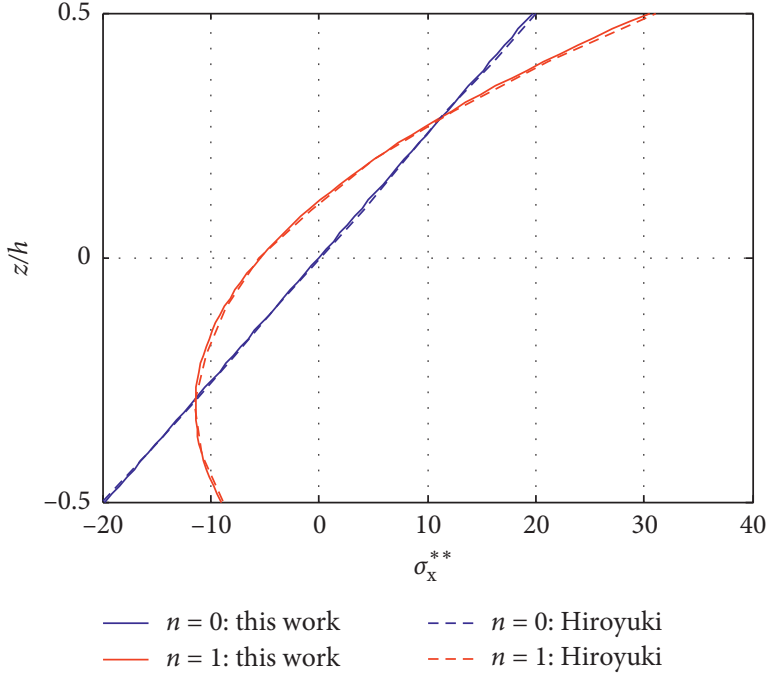

(b)

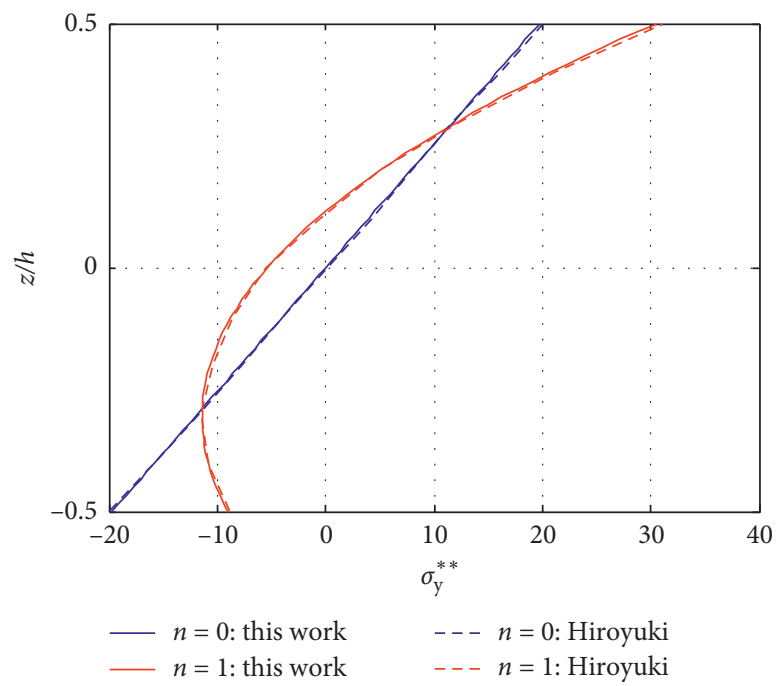

(c)

Figure 3: The comparison of the stress distribution of FGM subjected to bisinusoidal load: (a) $\tau_{x y}^{* *}(0,0, z) ;(\mathrm{b}) \sigma_{x}^{* *}((a / 2),(b / 2), z)$; (c) $\sigma_{y}^{* *}((a / 2),(b / 2), z)$.

Table 3: Nondimensional deflection of fully simply supported homologous nanoplate subjected to uniformly distributed load.

\begin{tabular}{lcccc}
\hline$\psi$ & & $b / a=1$ & & $b / a=2$ \\
[33] & This work & 0.7169 & 0.7144 \\
\hline 0 & 4.1853 & 4.2732 & 0.8767 & 0.8567 \\
0.5 & 4.5607 & 4.6416 & 1.0364 & 0.9991 \\
1 & 4.9362 & 5.0100 & 1.1961 & 1.1415 \\
1.5 & 5.3116 & 5.3784 & 1.3558 & 1.2838 \\
2 & 5.6871 & 5.7468 & 1.5155 & 1.4262 \\
2.5 & 6.0625 & 6.1152 & 1.6752 & 1.5685 \\
3 & 6.4380 & 6.4836 & & \\
\hline
\end{tabular}

distinctly different as the difference of the maximum value $\tau_{x y}^{*}$.

(iii) For the CFFF plate, the maximum values of all stresses $\sigma_{x}^{*}, \sigma_{y}^{*}, \tau_{x y}^{*}, \tau_{x z}^{*}$, and $\tau_{y z}^{*}$ are greater than those of the SSSS, CCCC, and CFCF plates. For all cases of the CCCC plate, there is a position that the stresses $\sigma_{x}^{*}, \sigma_{y}^{*}, \tau_{x y}^{*}, \tau_{x z}^{*}$, and $\tau_{y z}^{*}$ are zero; however, these positions are different for each particular 

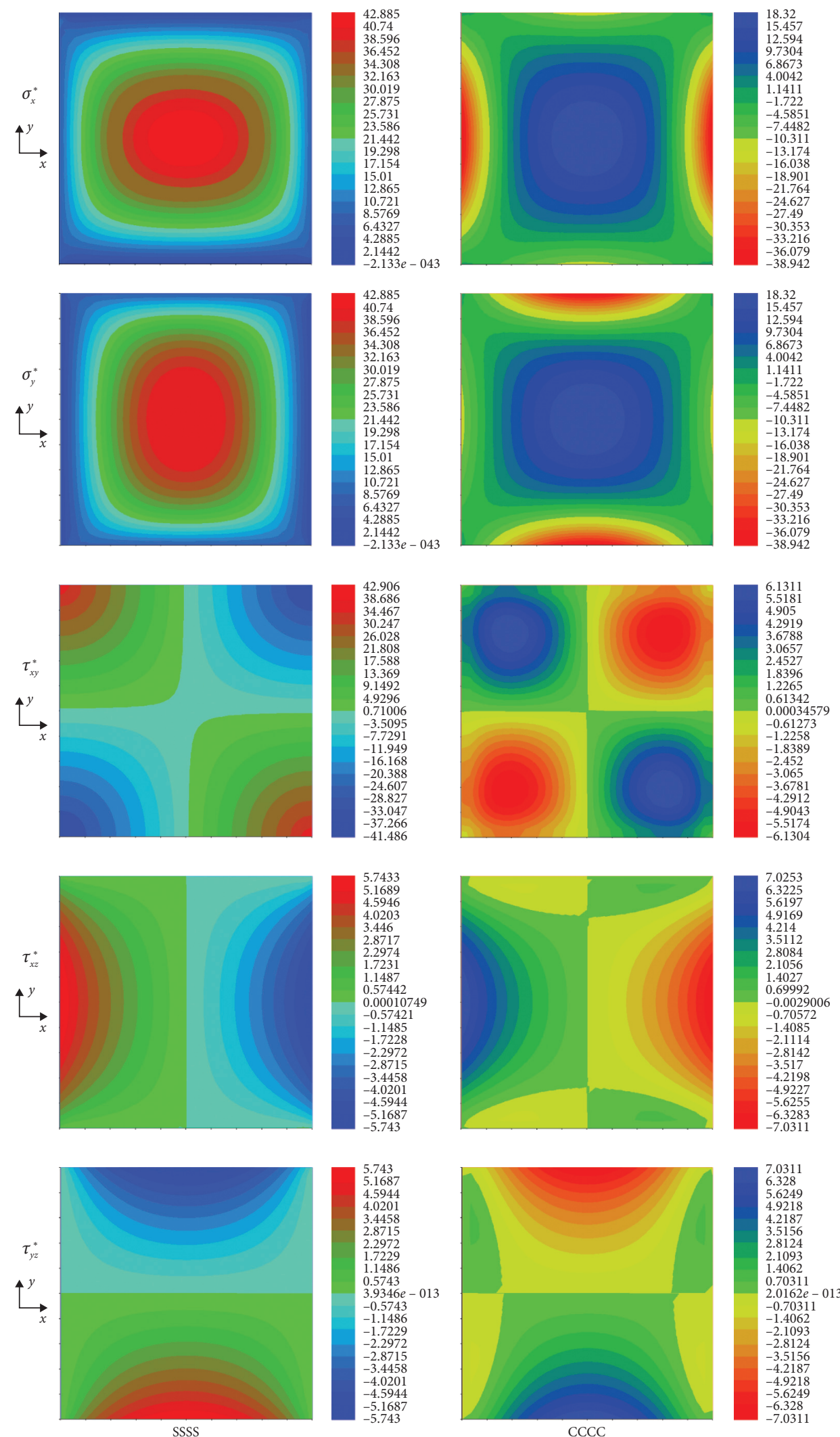

FIGURE 4: The stress distribution surface ( $x y$-plane) of FGM nanoplate subjected to uniformly distributed load $q_{0}, n=1, a / h=10, a / b=1$, and $\psi=1$. 

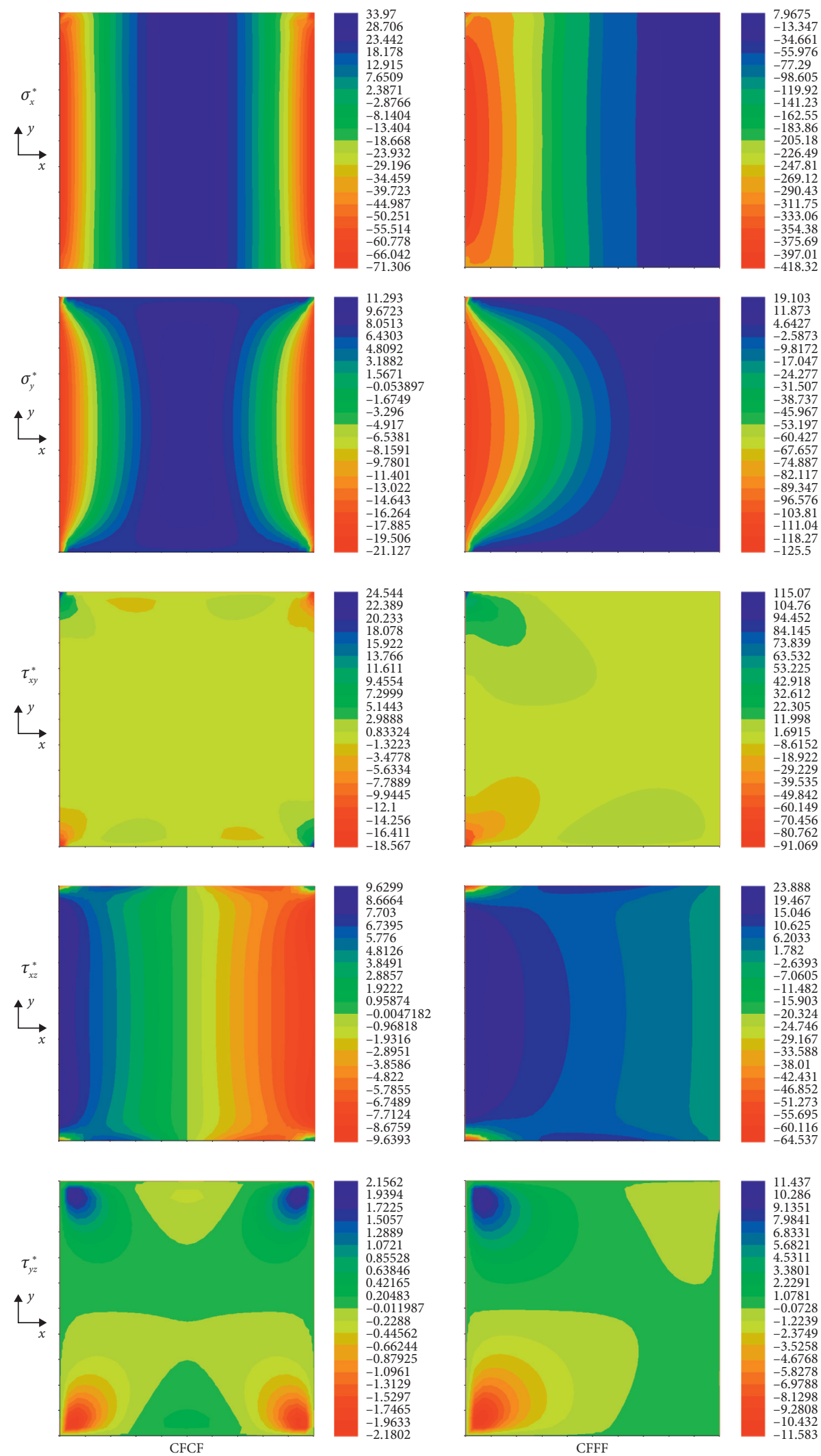

FIGURE 5: The stress distribution surface ( $x y$-plane) of FGM nanoplate subjected to uniformly distributed load $q_{0}, n=1, a / h=10, a / b=1$, and $\psi=1$. 

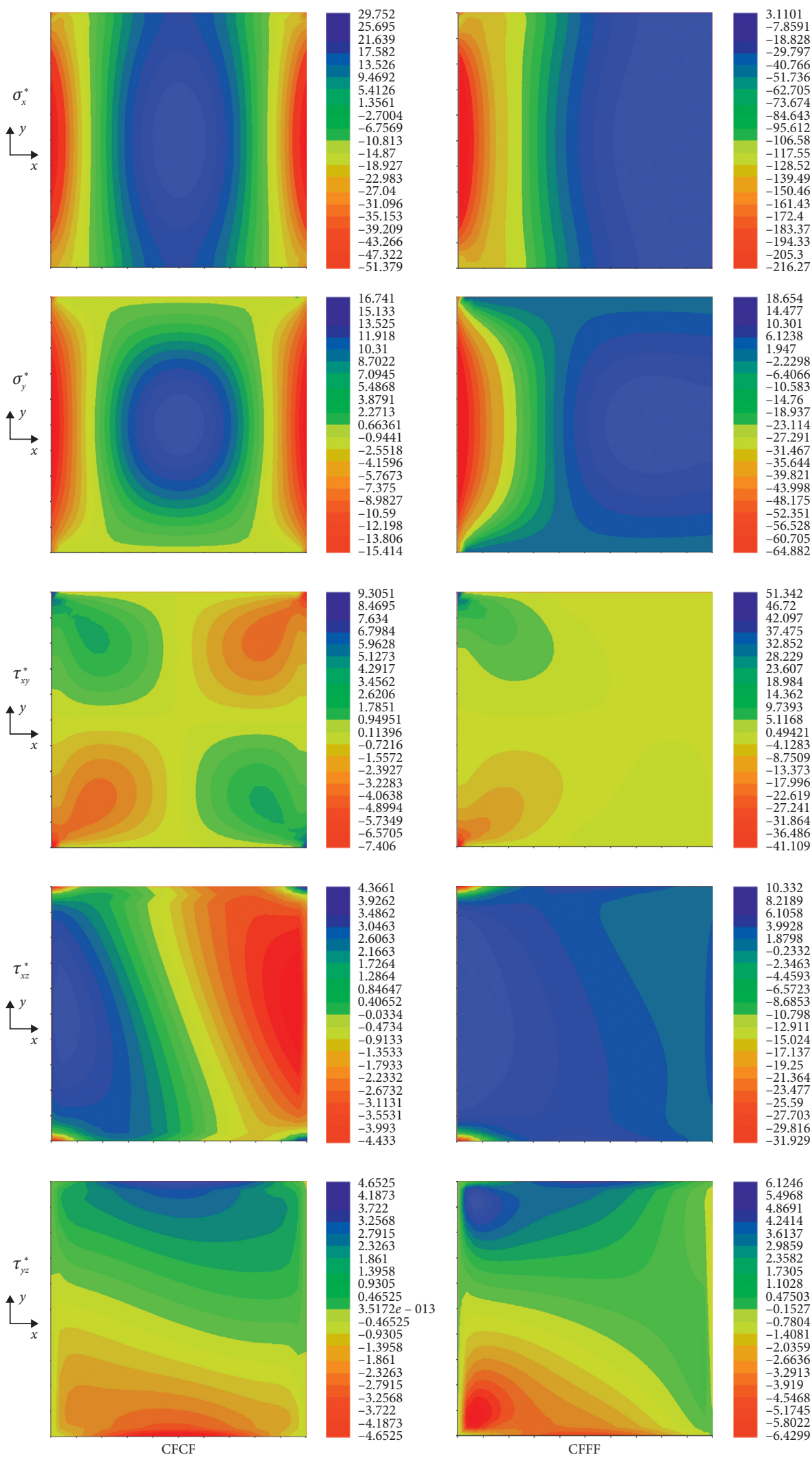

FIGURE 6: The stress distribution surface ( $x y$-plane) of FGM nanoplate subjected to sinusoidally distributed load $n=1, a / h=10, a / b=1$, and $\psi=1$. 
TABLE 4: The maximum values of deflection and stress of FGM nanoplate subjected to uniformly distributed load $q_{0}, n=1, a / h=10, a / b=1$, and $\psi=1$.

\begin{tabular}{lcccc}
\hline & & \multicolumn{3}{c}{ Boundary condition } \\
& SSSS & CCCC & CFCF & CFFF \\
\hline$\left|w^{*}\right|_{\max }$ & 8.204 & 2.448 & 4.934 & 208.730 \\
$\left|\sigma_{x}^{*}\right|_{\max }$ & 42.885 & 38.942 & 71.300 & 418.320 \\
$\left|\sigma_{y}^{*}\right|_{\max }$ & 42.885 & 38.942 & 21.120 & 125.500 \\
$\left|\tau_{x y}^{*}\right|_{\max }$ & 42.906 & 6.131 & 24.540 & 115.070 \\
$\left|\tau_{x z}^{*}\right|_{\max }$ & 5.743 & 7.0311 & 9.629 & 64.537 \\
$\left|\tau_{y z}^{*}\right|_{\max }$ & 5.743 & 7.0311 & 2.802 & 11.585 \\
\hline
\end{tabular}

TABLE 5: The maximum values of deflection and stress of FGM nanoplate subjected to sinusoidally distributed load $q_{0}, n=1, a / h=10, a / b=1$, and $\psi=1$.

\begin{tabular}{lcccc}
\hline & & & Boundary condition & \\
& SSSS & CCCC & CFCF & CFFF \\
\hline$\left|w^{*}\right|_{\max }$ & 6.144 & 2.402 & 3.912 & 95.890 \\
$\left|\sigma_{x}^{*}\right|_{\max }$ & 36.239 & 33.932 & 51.379 & \\
$\left|\sigma_{y}^{*}\right|_{\max }$ & 36.239 & 33.844 & 16.741 & \\
$\left|\tau_{x y}^{*}\right|_{\max }$ & 19.506 & 6.112 & 9.305 & 64.882 \\
$\left|\tau_{x z}^{*}\right|_{\max }$ & 1.964 & 3.160 & 4.433 & 51.342 \\
$\left|\tau_{y z}^{*}\right|_{\max }$ & 0.745 & 1.715 & 4.652 & 31.929 \\
\hline
\end{tabular}
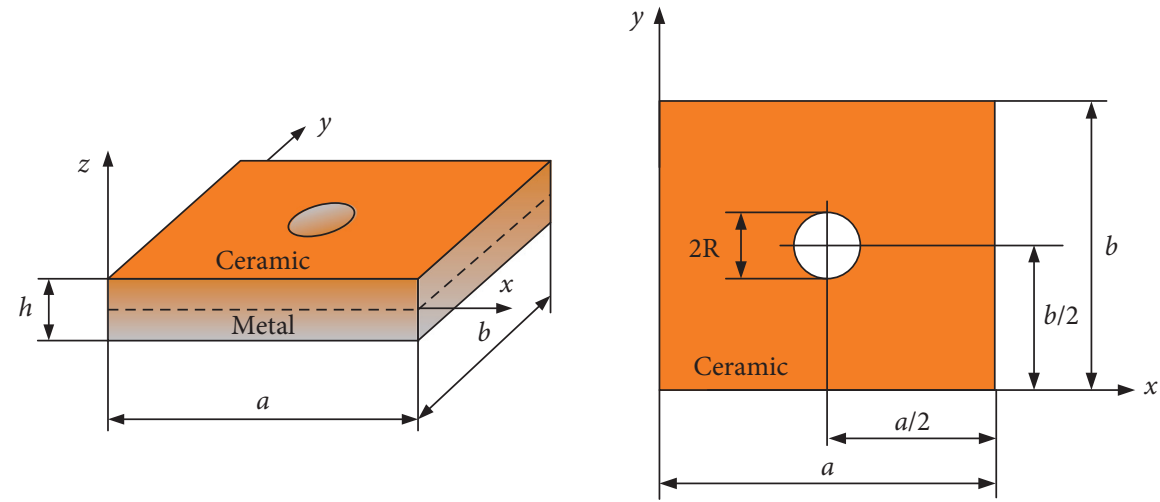

FIGURE 7: An FGM nanoplate with a circle hole.

plate. The calculation results also show that the maximum stresses of all stress components for CFCF and CFFF plates are located in the middle points of clamped edges and around the corners of the plates. This demonstrates that plates under these boundary conditions are susceptible to damage at the clamped edges, and these positions should be preferred to test for their strength during working processes.

(iv) For the same uniformly distributed load $q_{0}$, the maximum displacement and stress of the plate under uniformly distributed load are much greater than those of the sinusoidally distributed load.

(v) The numerical results also confirm that the position with the largest deflection $w_{\max }$ does not coincide with the position with the maximum values of the stress, except for the case of the SSSS plate.
5.2. The Stress Distribution of FGM Nanoplate with a Circle Hole. Consider a square FGM nanoplate with a circle hole (the radius of the circle hole $R=a / 10$ ) in the center of the plate as shown in Figure 7 . The plate is under a uniformly distributed load $q_{0}$.

5.2.1. Effect of the Hole. Figure 8 presents the stress distribution in the longitudinal directions of edges for the SSSS and CCCC plates. Table 6 shows the maximum deflection and stress of plates with different boundary conditions. The numerical results indicate that the CCCC plate with and without a hole has the same position as the maximum stress. However, for the SSSS plate with and without a hole, the maximum stresses $\sigma_{x}^{*}$ and $\sigma_{y}^{*}$ appear in the rear area of the hole. The maximum values of deflection and stress of the CFFF plate are much higher than those of other boundary conditions. 

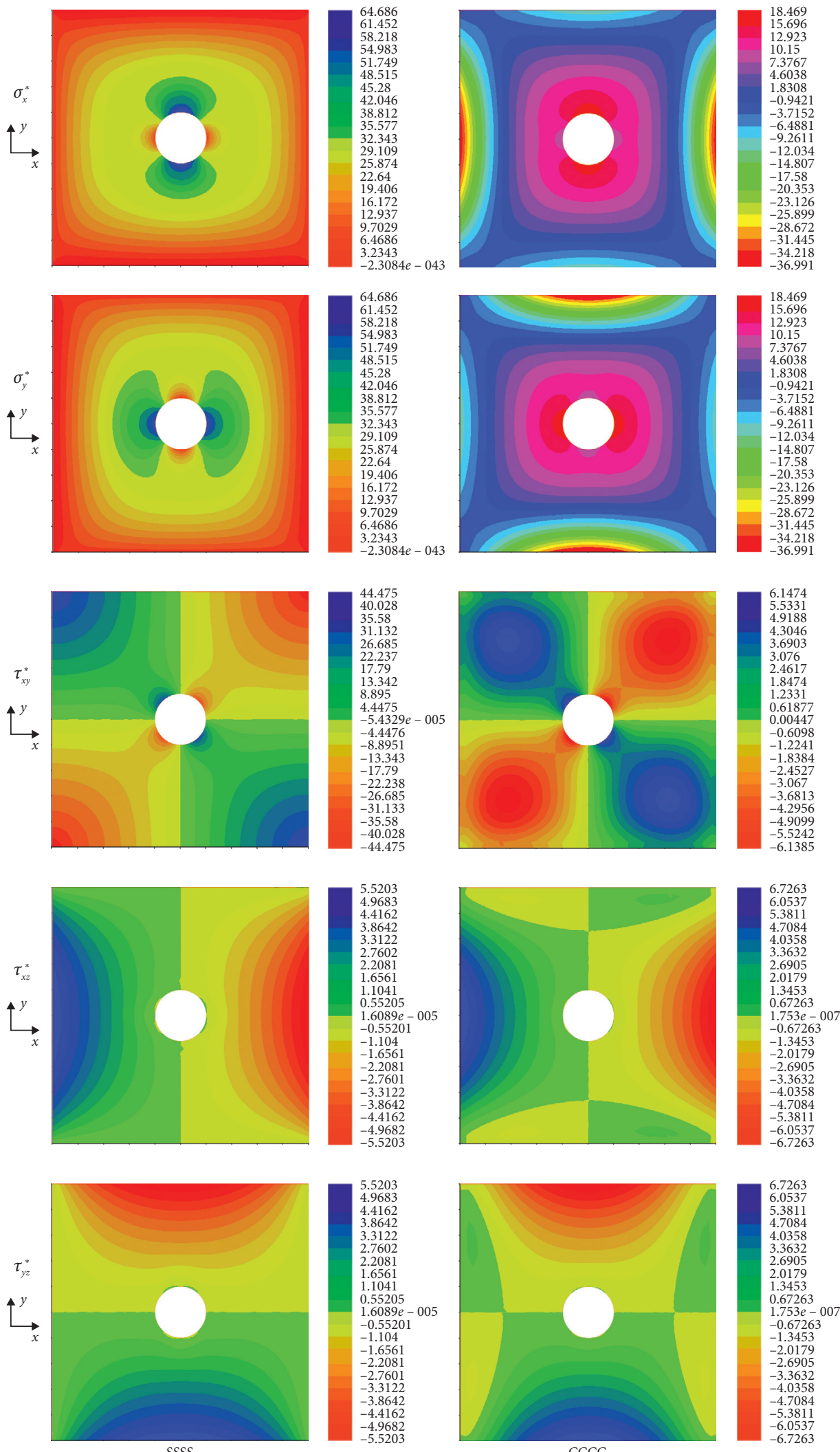

FIGURE 8: The stress distribution surface along the edges of the plate subjected to uniformly distributed load, $n=1, a / h=10, a / b=1$, and $\psi=1$. 
TABLE 6: Maximum deflection and stress of FGM nanoplate with a hole $(R=a / 10)$ subjected to uniformly distributed load, $n=1, a$ / $h=10, a / b=1$, and $\psi=1$.

\begin{tabular}{lcccc}
\hline & \multicolumn{4}{c}{ Boundary condition } \\
& SSSS & CCCC & CFCF & CFFF \\
\hline$\left|w^{*}\right|_{\max }$ & 8.761 & 2.223 & 4.961 & 211.323 \\
$\left|\sigma_{x}^{*}\right|_{\max }$ & 64.686 & 36.990 & 76.070 & 404.569 \\
$\left|\sigma_{y}^{*}\right|_{\max }$ & 64.686 & 36.990 & 22.821 & 121.371 \\
$\left|\tau_{x y}^{*}\right|_{\max }$ & 44.475 & 6.147 & 26.410 & 119.865 \\
$\left|\tau_{x z}^{*}\right|_{\max }$ & 5.520 & 6.726 & 9.490 & 63.296 \\
$\left|\tau_{y z}^{*}\right|_{\max }$ & 5.520 & 6.726 & 4.117 & 52.180 \\
\hline
\end{tabular}

TABLE 7: The deflection $\left|w^{*}\right|_{\max }$ of fully simply supported FGM nanoplate, $a / h=10$, and $a / b=1$.

\begin{tabular}{lcccccc}
\hline$\psi$ & \multicolumn{7}{c}{$N$} \\
& 0 & 0.5 & 2 & 3 & 5 & 10 \\
\hline 0 & 5.153 & 6.804 & 8.566 & 8.933 & 9.364 & 10.072 \\
0.5 & 5.496 & 7.258 & 9.136 & 9.526 & 9.986 & 10.740 \\
1.0 & 5.839 & 7.712 & 9.706 & 10.120 & 10.607 & 11.407 \\
1.5 & 6.182 & 8.165 & 10.276 & 10.713 & 11.228 & 12.075 \\
2.0 & 6.525 & 8.619 & 10.846 & 11.307 & 11.849 & 12.743 \\
2.5 & 6.868 & 9.073 & 11.416 & 11.900 & 12.470 & 13.410 \\
3.0 & 7.210 & 9.527 & 11.986 & 12.494 & 13.091 & 14.078 \\
\hline
\end{tabular}

TABLE 8: Maximum stresses at $z=h / 2$ of fully simply supported FGM nanoplate subjected to uniformly distributed load, $a / h=10$, and $a / b=1$.

\begin{tabular}{cccccccc}
\hline & $n$ & & \multicolumn{6}{c}{$\psi$} \\
& & 0 & 0.5 & 1.0 & 1.5 & 2.0 & 2.5 \\
\hline & 0 & 48.298 & 49.056 & 49.814 & 50.593 & 51.350 & 52.106 \\
$\left|\sigma_{x}^{*}\right|_{\max }$ & 0.5 & 57.740 & 58.645 & 59.550 & 60.456 & 61.361 & 62.267 \\
& 2 & 67.767 & 68.828 & 69.890 & 70.951 & 72.013 & 73.074 \\
& 10 & 85.703 & 87.042 & 88.381 & 89.720 & 91.059 & 92.398 \\
\hline & 0 & 48.298 & 49.056 & 49.814 & 50.503 & 51.350 & 52.126 \\
$\left|\sigma_{y}^{*}\right|_{\max }$ & 0.5 & 57.740 & 58.585 & 59.550 & 60.456 & 61.361 & 62.267 \\
& 2 & 67.767 & 68.828 & 69.890 & 70.951 & 72.013 & 73.074 \\
& 10 & 85.703 & 87.042 & 88.381 & 89.720 & 91.059 & 92.398 \\
\hline & 0 & 25.456 & 27.260 & 34.260 & 41.260 & 48.259 & 55.259 \\
$\left|\tau_{x y}^{*}\right|_{\max }$ & 0.5 & 30.401 & 32.564 & 40.926 & 49.287 & 57.649 & 66.010 \\
& 2 & 35.696 & 38.228 & 48.403 & 57.859 & 67.675 & 77.490 \\
& 10 & 45.185 & 48.368 & 60.787 & 73.207 & 85.626 & 98.045 \\
\hline \multirow{4}{*}{$\left|\tau_{x z}^{*}\right|_{\max }$} & 0 & 3.893 & 3.894 & 3.895 & 3.896 & 3.897 & 3.898 \\
& 0.5 & 4.852 & 4.853 & 4.854 & 4.855 & 4.856 & 4.857 \\
& 2 & 6.401 & 6.402 & 6.403 & 6.404 & 6.405 & 6.406 \\
& 10 & 8.344 & 8.345 & 8.346 & 8.347 & 8.348 & 8.349 \\
\hline & 0 & 3.893 & 3.894 & 3.895 & 3.896 & 3.897 & 3.898 \\
$\left|\tau_{y z}^{*}\right|_{\max }$ & 0.5 & 4.852 & 4.853 & 4.854 & 4.855 & 4.856 & 4.857 \\
& 2 & 6.401 & 6.402 & 6.403 & 6.404 & 6.405 & 6.406 \\
& 10 & 8.344 & 8.345 & 8.346 & 8.347 & 8.348 & 8.349 \\
\hline
\end{tabular}

5.2.2. Effect of Volume Fraction Exponent $n$. Consider a fully simply supported FGM nanoplate subjected to uniformly distributed load. Let the volume fraction exponent $n$ varies in a range of 0 to 10 , and the maximum value of deflection $\left|w^{*}\right|_{\max }$ and stress components changing as a function of $n$ and $\psi$ are shown in Tables 7 and 8. It can be seen that when the volume fraction exponent $n$ increases, both maximum deflection and stress increase. In addition, when $\psi$ increases, the distance between molecules increases, the plate also becomes softer, and therefore the maximum deflection and stress increase.

\section{Conclusions}

This paper presents new numerical results of stress distributions along the edges of FGM nanoplates. The finite element formulations are derived from the first-order shear deformation theory of Mindlin; the proposed theory and mathematical model are verified by comparing the numerical data with those of published results. Some new main points of this work are concluded as follows:

(i) Boundary condition and the shape of the plate have a strong effect on the maximum value of stress as well as the positions of maximum stress. For the plate without a hole, the stresses $\sigma_{x}^{*}$ and $\sigma_{y}^{*}$ obtain the maximum values at the center point of the SSSS plate. In contrast, for the CCCC plate, these stresses obtain the maximum values at the boundaries. Nevertheless, for the plate with a hole, the CCCC plate has the same position as the maximum stress; for the SSSS plate, the maximum stresses $\sigma_{x}^{*}$ and $\sigma_{y}^{*}$ appear in the rear area of the hole. This is an important suggestion for scientists when considering the strengths of FGM nanoplates to pay close attention to the positions with maximum stress.

(ii) The fewer degree of freedom of the structure is constrained; the greater the maximum stress value, the maximum stress position usually appears around clamped regions.

(iii) When increasing the value of $\psi$, for any values of the volume fraction exponent $n$, deflection and stress are increased due to the increase in the distance between molecules.

\section{Data Availability}

The data used to support the findings of this study are included in the article.

\section{Conflicts of Interest}

The author declares that there are no conflicts of interest regarding the publication of this paper.

\section{Acknowledgments}

This work was supported by the University of Transport Technology Foundation for Science and Technology Development (grant no. 1139/QD).

\section{References}

[1] T. Q. Bui, T. D. Do, L. T. T. Ton et al., "On the high temperature mechanical behaviors analysis of heated functionally graded plates using FEM and a new third-order shear 
deformation plate theory," Composites Part B: Engineering, vol. 92, pp. 218-241, 2016.

[2] M. D. Demirbas, "Thermal stress analysis of functionally graded plates with temperature-dependent material properties using theory of elasticity," Composites Part B: Engineering, vol. 131, pp. 100-124, 2017.

[3] M. Adineh and M. Kadkhodayan, "Three-dimensional thermo-elastic analysis and dynamic response of a multidirectional functionally graded skew plate on elastic foundation," Composites Part B: Engineering, vol. 125, pp. 227-240, 2017.

[4] T. V. Do, D. K. Nguyen, N. D. Duc, H. D. Duc, and Q. B. Tinh, "Analysis of bi-directional functionally graded plates by FEM and a new third-order shear deformation plate theory," ThinWalled Structures, vol. 119, pp. 687-699, 2017.

[5] S. A. Yahia, L. H. H. Amar, Z. Belabed, and A. Tounsi, "Effect of homogenization models on stress analysis of functionally graded plates," Structural Engineering and Mechanics, vol. 67, no. 5, pp. 527-544, 2018.

[6] M. Hosseini, M. Shishesaz, K. N. Tahan, and A. Hadi, "Stress analysis of rotating nano-disks of variable thickness made of functionally graded materials," International Journal of Engineering Science, vol. 109, pp. 29-53, 2016.

[7] D. Shahriar and B. Akgöz, "New static and dynamic analyses of macro and nano FGM plates using exact three-dimensional elasticity in thermal environment," Composite Structures, vol. 192, pp. 626-641, 2018.

[8] R. Ansari, J. Torabi, and A. Norouzzadeh, "Bending analysis of embedded nanoplates based on the integral formulation of Eringen's nonlocal theory using the finite element method," Physica B: Condensed Matter, vol. 534, pp. 90-97, 2018.

[9] B. Babu and B. P. Patel, "A new computationally efficient finite element formulation for nanoplates using second-order strain gradient Kirchhoff's plate theory," Composites Part B: Engineering, vol. 168, pp. 302-311, 2019.

[10] M. Repka, V. Sladek, and J. Sladek, "Numerical study of size effects in micro/nano plates by moving finite elements," Composite Structures, vol. 212, pp. 291-303, 2019.

[11] C. H. Thai, A. J. M. Ferreira, and P. Phung-Van, "A nonlocal strain gradient isogeometric model for free vibration and bending analyses of functionally graded plates," Composite Structures, vol. 251, Article ID 112634, 2020.

[12] R. Ansari, R. Hassani, R. Gholami, and H. Rouhi, "Nonlinear bending analysis of arbitrary-shaped porous nanocomposite plates using a novel numerical approach," International Journal of Non-linear Mechanics, vol. 126, Article ID 103556, 2020.

[13] F. Heidari, K. Taheri, M. Sheybani, and M. Janghorban, "On the mechanics of nanocomposites reinforced by wavy/ defected/aggregated nanotubes," Steel and Composite Structures, vol. 38, no. 5, pp. 533-545, 2021.

[14] A. Rouabhia, A. Chikh, A. A. Bousahla et al., "Physical stability response of a SLGS resting on viscoelastic medium using nonlocal integral first-order theory," Steel and Composite Structures, vol. 37, no. 6, pp. 695-709, 2020.

[15] H. Matouk, A. A. Bousahl, H. Heireche et al., "Investigation on hygro-thermal vibration of P-FG and symmetric S-FG nanobeam using integral Timoshenko beam theory," Advances in Nano Research, vol. 8, no. 4, pp. 293-305, 2020.

[16] M. Bellal, H. Hebali, H. Heireche et al., "Buckling behavior of a single-layered graphene sheet resting on viscoelastic medium via nonlocal four-unknown integral model," Steel and Composite Structures, vol. 34, no. 5, pp. 643-655, 2020.
[17] S. Asghar, M. N. Naeem, M. Hussain, M. Taj, and A. Tounsi, "Prediction and assessment of nonlocal natural frequencies of DWCNTs: vibration analysis," Computers and Concrete, vol. 25, no. 2, pp. 133-144, 2020.

[18] M. Balubaid, A. Tounsi, B. Dakhel, and S. R. Mahmoud, "Free vibration investigation of FG nanoscale plate using nonlocal two variables integral refined plate theory," Computers and Concrete, vol. 24, no. 6, pp. 579-586, 2019.

[19] H. Hachemi, A. A. Bousahla, K. Kaci et al., "Bending analysis of functionally graded plates using a new refined quasi-3D shear deformation theory and the concept of the neutral surface position," Steel and Composite Structures, vol. 39, no. 1, pp. 051-064, 2021.

[20] A. Bakoura, F. Bourada, A. A. Bousahla et al., "Buckling analysis of functionally graded plates using HSDT in conjunction with the stress function method," Computers and Concrete, vol. 27, no. 1, pp. 73-83, 2021.

[21] A. Menasria, A. Kaci, A. A. Bousahla et al., "A four-unknown refined plate theory for dynamic analysis of FG-sandwich plates under various boundary conditions," Steel and Composite Structures, vol. 36, no. 3, pp. 355-367, 2020.

[22] J. N. Reddy, "Analysis of functionally graded plates," International Journal for Numerical Methods in Engineering, vol. 47, no. 1-3, pp. 663-684, 2000.

[23] H. N. Nguyen, T. C. Tan, D. T. Luat, P. V. Duc, D. V. Thom, and P. V. Minh, "Research on the buckling behavior of functionally graded plates with stiffeners based on the thirdorder shear deformation theory," Materials, vol. 12, no. 8, Article ID 1262, 2019.

[24] D. V. Thom, D. H. Duc, P. V. Minh, and N. S. Tung, "Finite element modelling for free vibration response of cracked stiffened FGM plates," Vietnam Journal Of Science And Technology, vol. 58, no. 1, p. 119, 2020.

[25] A. C. Eringen, "On differential equations of nonlocal elasticity and solutions of screw dislocation and surface waves," Journal of Applied Physics, vol. 54, no. 9, pp. 4703-4710, 1983.

[26] A. C. Eringen, Nonlocal Continuum Field Theories, Springer, Manhattan, NY, USA, 2002.

[27] L. Li, R. Lin, and Y. Hu, "Cross-section effect on mechanics of nonlocal beams," Archive of Applied Mechanics, vol. 91, no. 4, pp. 1541-1556, 2021.

[28] L. Li, R. Lin, and T. Y. Ng, "Contribution of nonlocality to surface elasticity," International Journal of Engineering Science, vol. 152, Article ID 103311, 2020.

[29] V. D. Thom, H. D. Duc, D. D. Nguyen, and Q. B. Tinh, "Phasefield thermal buckling analysis for cracked functionally graded composite plates considering neutral surface," Composite Structures, vol. 182, pp. 542-548, 2017.

[30] A. M. Zenkour, "Generalized shear deformation theory for bending analysis of functionally graded plates," Applied Mathematical Modelling, vol. 30, no. 1, pp. 67-84, 2006.

[31] M. K. Singha, T. Prakash, and M. Ganapathi, "Finite element analysis of functionally graded plates under transverse load," Finite Elements in Analysis and Design, vol. 47, no. 4, pp. 453-460, 2011.

[32] M. Hiroyuki, "Stress analysis of functionally graded plates subjected to thermal and mechanical loadings," Composite Structures, vol. 87, no. 4, pp. 344-357, 2009.

[33] R. Aghababaei and J. N. Reddy, "Nonlocal third-order shear deformation plate theory with application to bending and vibration of plates," Journal of Sound and Vibration, vol. 326, no. 1-2, pp. 277-289, 2009. 